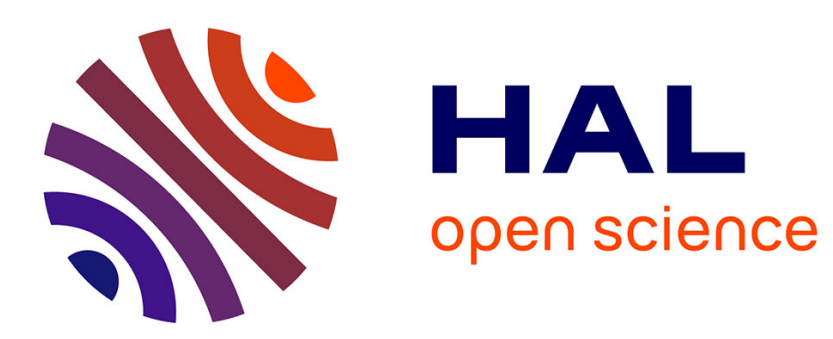

\title{
Minimum stress optimal design with the level set method
}

Grégoire Allaire, François Jouve

\section{To cite this version:}

Grégoire Allaire, François Jouve. Minimum stress optimal design with the level set method. Engineering Analysis with Boundary Elements, 2008, 32, pp.909-918. hal-01089075

\section{HAL Id: hal-01089075 \\ https://hal.science/hal-01089075}

Submitted on 4 Feb 2020

HAL is a multi-disciplinary open access archive for the deposit and dissemination of scientific research documents, whether they are published or not. The documents may come from teaching and research institutions in France or abroad, or from public or private research centers.
L'archive ouverte pluridisciplinaire HAL, est destinée au dépôt et à la diffusion de documents scientifiques de niveau recherche, publiés ou non, émanant des établissements d'enseignement et de recherche français ou étrangers, des laboratoires publics ou privés. 


\title{
Minimum stress optimal design with the level set method
}

\author{
Grégoire ALLAIRE, \\ CMAP, ECOLE POLYTECHNIQUE, CNRS, \\ 91128 Palaiseau, France \\ and François JOUVE, \\ Laboratoire J.L.Lions, Université Paris 7 - Denis Diderot \\ 75252 Paris, France
}

May 29, 2007

\begin{abstract}
This paper is devoted to minimum stress design in structural optimization. We propose a simple and efficient numerical algorithm for shape and topology optimization based on the level set method coupled with the topological derivative. We compute a shape derivative, as well as a topological derivative, for a stress-based objective function. Using an adjoint equation we implement a gradient algorithm for the minimization of the objective function. Several numerical examples in 2-d and 3-d are discussed.
\end{abstract}

\section{Introduction}

Since the seminal papers [6], [7], [27], [34], [40], there has been a burst of publications on the application of the level set method to shape and topology optimization of structures. Most of the recent papers focus on numerical issues for improving the level set method but do not extend so much its range of applicability. Let us mention, for example, works on its coupling with the topological gradient for holes nucleation [3], [13], [41], or velocity/derivative regularization [20]. Most of these recent works consider only compliance optimization which is a notably simpler problem than optimization of a general objective function. The goal of the present paper is, on the contrary, to extend the range of objective functions which are successfully treated by the level set method, and more specifically to treat the case of objective functions depending on the stress tensor. Together with our previous works [4] (for eigenvalue and multiple loads optimization) and [21] (for robust or worst-case optimization) it clearly demonstrates that the level set method is a versatile tool for structural optimization which can tackle industrial, and not merely academic, problems. 
Stress objective functions have been studied for a long time in the classical setting of geometric shape optimization (in the framework of Hadamard method [24], [31], [38]). There are relatively fewer papers on this topic in the context of topology optimization, based either on the homogenization method, SIMP or phase-field method, see [5], [17], [16], [23], [39]. There are however a vast literature on truss optimization with stress constraints or stress-based objective functions, see e.g. [1], [30]. In any case, there are still simple test problems which are not satisfactorily handled by topology optimization methods (see Section 6). Therefore, it is important to prove by numerical evidence that the level set method is a viable approach for this type of problems.

The content of the paper is the following. In Section 2 the problem of minimum stress design is introduced in the classical setting of shape optimization. Section 3 is devoted to computing the shape derivative of the stress-based objective function using an adjoint method. Section 4 recalls the necessary ingredients on the level set method of Osher and Sethian [28], [26], [33]. Section 5 gives the details of the proposed numerical algorithm and discusses numerical examples.

\section{Setting of the problem}

In this paper we restrict ourselves to linear elasticity although there is no conceptual difficulty in extending our work to non-linear elasticity (see [7]). A shape is a bounded open set $\Omega \subset \mathbb{R}^{d}(d=2$ or 3 ) with a boundary made of three disjoint parts

$$
\partial \Omega=\Gamma \cup \Gamma_{N} \cup \Gamma_{D},
$$

where only $\Gamma$ is subject to optimization and free to move, while $\Gamma_{N}$ and $\Gamma_{D}$ are fixed. Homogeneous Neumann boundary condition (no traction) is imposed on the free boundary $\Gamma$, a Dirichlet boundary condition on $\Gamma_{D}$ and a Neumann boundary condition on $\Gamma_{N}$. All admissible shapes $\Omega$ are required to be a subset of a working domain $D$ (a bounded open set of $\mathbb{R}^{d}$ ). The shape $\Omega$ is occupied by a linear isotropic elastic material with Hooke's law $A$ defined, for any symmetric matrix $\xi$, by

$$
A \xi=2 \mu \xi+\lambda(\operatorname{Tr} \xi) I_{2},
$$

where $\mu$ and $\lambda$ are the Lamé moduli of the material. The displacement field $u$ in $\Omega$ is the solution of the linearized elasticity system

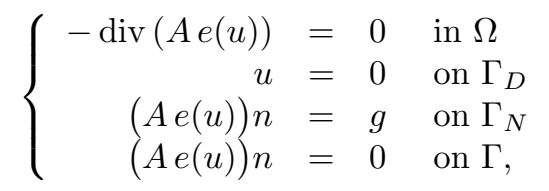

where $g \in H^{-1 / 2}\left(\Gamma_{N}\right)^{d}$ is a given surface load. For simplicity we do not consider volume forces here although there is again no difficulty to take them into account (see [7]). Assuming that the surface measure of $\Gamma_{D}$ is not zero (otherwise we 
should impose an equilibrium condition on $g),(2)$ admits a unique solution in $u \in H^{1}(\Omega)^{d}$. is

The objective function is denoted by $J(\Omega)$. A typical example in this paper

$$
J(\Omega)=\int_{\Omega} k(x)|\sigma|^{2} d x
$$

where $k(x) \in L^{\infty}(D)$ is a given piecewise smooth non-negative function (a weighting factor that can localize the objective function). More generally we can set

$$
J(\Omega)=\int_{\Omega} j(x, \sigma(x)) d x,
$$

with a smooth function $j$. This allows us, for example, to minimize the equivalent Von Mises stress intensity in $\Omega$, or to reach a stress target $\sigma_{0}$ (a useful criterion for mechanism design). Similarly, we could consider a function $j(x, e(u)(x))$ depending on the strain tensor. In both formulas (3) and (4), the stress tensor is

$$
\sigma=A e(u)
$$

where $u=u(\Omega)$ is the solution of (2). We define the set of admissible shapes that must be open sets contained in the working domain $D$, of fixed volume $V$ and satisfying (1)

$$
\mathcal{U}_{a d}=\left\{\Omega \subset D \text { such that }|\Omega|=V, \Gamma_{N} \cup \Gamma_{D} \subset \partial \Omega\right\} .
$$

Our model problem of shape optimization is

$$
\inf _{\Omega \in \mathcal{U}_{a d}} J(\Omega) .
$$

In practice we often work with an unconstrained problem. Introducing a Lagrange multiplier $\ell$, we consider the Lagrangian minimization

$$
\inf _{\Omega \in \mathcal{U}_{a d}} \mathcal{L}(\Omega)=J(\Omega)+\ell|\Omega| .
$$

\section{Shape derivative}

In order to apply a gradient method to the minimization of (6) we recall the classical notion of shape derivative, going back to Hadamard (see e.g. [2], [24], [31], [38]). Starting from a smooth reference open set $\Omega$, we consider domains of the type

$$
\Omega_{\theta}=\left(I_{2}+\theta\right)(\Omega),
$$

with $I_{2}$ the identity mapping from $\mathbb{R}^{d}$ into $\mathbb{R}^{d}$ and $\theta$ a vector field in $W^{1, \infty}\left(\mathbb{R}^{d}, \mathbb{R}^{d}\right)$. It is well known that, for sufficiently small $\theta,\left(I_{2}+\theta\right)$ is a diffeomorphism in $\mathbb{R}^{d}$. We remark that all admissible domains $\Omega_{\theta}$ belong to the class of homotopy of the reference domain $\Omega$ (it implies that in 2 -d the number of connected components of the boundary remains constant). In other words, no change of topology is possible with this method of shape variation. 
Definition 3.1. The shape derivative of $J(\Omega)$ at $\Omega$ is defined as the Fréchet derivative in $W^{1, \infty}\left(\mathbb{R}^{d}, \mathbb{R}^{d}\right)$ at 0 of the application $\theta \rightarrow J\left(\left(I_{2}+\theta\right)(\Omega)\right)$, i.e.

$$
J\left(\left(I_{2}+\theta\right)(\Omega)\right)=J(\Omega)+J^{\prime}(\Omega)(\theta)+o(\theta) \quad \text { with } \quad \lim _{\theta \rightarrow 0} \frac{|o(\theta)|}{\|\theta\|}=0,
$$

where $J^{\prime}(\Omega)$ is a continuous linear form on $W^{1, \infty}\left(\mathbb{R}^{d}, \mathbb{R}^{d}\right)$.

Because of the constraint (1) on the boundary of all admissible shapes, we always assume that the vector fields $\theta$ vanish on $\Gamma_{N}$ and $\Gamma_{D}$, which ensures that $\Omega_{\theta}$ satisfy (1). Let us recall the following classical lemma (see e.g. [2], [24], [31], $[38])$.

Lemma 3.2. Let $\Omega \in \mathcal{U}_{a d}$ be a smooth bounded open set and $\phi(x)$ a smooth function defined in $\mathbb{R}^{d}$. Define

$$
J_{v o l}(\Omega)=\int_{\Omega} \phi(x) d x \quad \text { and } \quad J_{\text {surf }}(\Omega)=\int_{\partial \Omega} \phi(x) d s .
$$

These two functions are shape differentiable at $\Omega \in \mathcal{U}_{\text {ad }}$ and

$$
\begin{gathered}
J_{v o l}^{\prime}(\Omega)(\theta)=\int_{\Gamma} \theta \cdot n \phi d s, \\
J_{\text {surf }}^{\prime}(\Omega)(\theta)=\int_{\Gamma} \theta \cdot n\left(\frac{\partial \phi}{\partial n}+H \phi\right) d s,
\end{gathered}
$$

for any $\theta \in W^{1, \infty}\left(\mathbb{R}^{d} ; \mathbb{R}^{d}\right)$ vanishing on $\Gamma_{N} \cup \Gamma_{D}$, where $H$ is the mean curvature of $\partial \Omega$ defined by $H=\operatorname{div} \tilde{n}$ (where $\tilde{n}$ is a local extension of the normal $n$ near $\Gamma)$.

Our main result in this section is the following theorem.

Theorem 3.3. Let $\Omega \in \mathcal{U}_{a d}$ be a smooth bounded open set and $\theta \in W^{1, \infty}\left(\mathbb{R}^{d} ; \mathbb{R}^{d}\right)$. Assume that the data $g$ as well as the solution $u$ of (2) are smooth, say $g \in$ $H^{2}(\Omega)^{d}, u \in H^{2}(\Omega)^{d}$. The shape derivative of (4) is

$$
J^{\prime}(\Omega)(\theta)=\int_{\Gamma} \theta \cdot n(j(x, \sigma)+A e(u) \cdot e(p)) d s,
$$

where $\sigma=A e(u)$ and $p$ is the adjoint state, assumed to be smooth, say $p \in$ $H^{2}(\Omega)^{d}$, defined as the solution of

$$
\left\{\begin{aligned}
-\operatorname{div}(A e(p)) & =\operatorname{div}\left(A j^{\prime}(x, \sigma)\right) & & \text { in } \Omega \\
p & =0 & & \text { on } \Gamma_{D} \\
(A e(p)) n & =-\left(A j^{\prime}(x, \sigma)\right) n & & \text { on } \Gamma_{N} \cup \Gamma,
\end{aligned}\right.
$$

where $j^{\prime}$ denotes the partial derivative of $j(x, \sigma)$ with respect to $\sigma$. 
Proof. Although Theorem 3.3 is a standard result in the framework of shape variations, it has never been published in the above explicit form, so we indulge ourselves in giving its proof for the sake of completeness. To simplify the exposition, we give a short, albeit formal, proof based on the Lagrangian method ([14], [2]). To the general objective function (4) we associate the Lagrangian defined for $(v, q) \in\left(H^{1}\left(\mathbb{R}^{d} ; \mathbb{R}^{d}\right)\right)^{2}$, such that $v=q=0$ on $\Gamma_{D}$, by

$$
\mathcal{L}(\Omega, v, q)=\int_{\Omega} j(x, A e(v)(x)) d x+\int_{\Omega} A e(v) \cdot e(q) d x-\int_{\Gamma_{N}} q \cdot g d s .
$$

For notational simplicity we drop the $x$-dependence of the integrand $j$ in the sequel. In (11) $q$ is a Lagrange multiplier for the state equation and its boundary conditions. Note that the above Lagrangian $\mathcal{L}(\Omega, v, q)$ has nothing to do with that, denoted by $\mathcal{L}(\Omega)$, introduced in $(7)$. Recall that $\Gamma_{D}$ is fixed so there is no need to introduce a Lagrange multiplier for the Dirichlet boundary condition on $\Gamma_{D}$. The two functions $v$ and $q$ belong to a functional space that does not depend on $\Omega \in \mathcal{U}_{a d}$, so we can apply the usual differentiation rule to the Lagrangian $\mathcal{L}$. The stationarity of the Lagrangian is going to give the optimality conditions of the minimization problem. For a given $\Omega$, we denote by $(u, p)$ such a stationary point. The partial derivative of $\mathcal{L}$ with respect to $q$, in the direction $\phi \in H^{1}\left(\mathbb{R}^{d} ; \mathbb{R}^{d}\right)$ satisfying $\phi=0$ on $\Gamma_{D}$, after integration by parts leads to

$$
\begin{aligned}
\left\langle\frac{\partial \mathcal{L}}{\partial q}(\Omega, u, p), \phi\right\rangle=0= & -\int_{\Omega} \phi \cdot(\operatorname{div}(A e(u))) d x \\
& +\int_{\Gamma_{N}} \phi \cdot((A e(u)) n-g) d s .
\end{aligned}
$$

Taking first $\phi$ with compact support in $\Omega$ gives the state equation. Then, varying the trace function $\phi$ on $\Gamma_{N}$ gives the Neumann boundary condition for $u$. On the other hand, in order to find the adjoint equation, we differentiate $\mathcal{L}$ with respect to $v$ in the direction $\phi \in H^{1}\left(\mathbb{R}^{d} ; \mathbb{R}^{d}\right)$ satisfying $\phi=0$ on $\Gamma_{D}$. This yields

$$
\left\langle\frac{\partial \mathcal{L}}{\partial v}(\Omega, u, p), \phi\right\rangle=0=\int_{\Omega} j^{\prime}(\sigma) \cdot A e(\phi) d x+\int_{\Omega} A e(\phi) \cdot e(p) d x .
$$

Integrating by parts we obtain

$$
\left.\frac{\partial \mathcal{L}}{\partial v}(\Omega, u, p), \phi\right\rangle=-\int_{\Omega} \operatorname{div}\left(A j^{\prime}(\sigma)+A e(p)\right) \cdot \phi d x+\int_{\Gamma_{N}} \phi \cdot A\left(e(p)+j^{\prime}(\sigma)\right) n d s .
$$

Taking first $\phi$ with compact support in $\Omega$ gives the adjoint state equation

$$
-\operatorname{div}(A e(p))=\operatorname{div}\left(A j^{\prime}(\sigma)\right) \quad \text { in } \Omega .
$$

Then, varying the trace of $\phi$ on $\Gamma_{N} \cup \Gamma$ yields the Neumann boundary condition

$$
(A e(p)) n=-\left(A j^{\prime}(\sigma)\right) n \quad \text { on } \quad \Gamma_{N} \cup \Gamma .
$$


Taking into account the constraint that $p=0$ on $\Gamma_{D}$ gives a well-posed boundary value problem for the adjoint state $p$.

The shape derivative of the objective function is obtained by differentiating

$$
J(\Omega)=\mathcal{L}(\Omega, u(\Omega), q),
$$

which yields

$$
J^{\prime}(\Omega)(\theta)=\left\langle\frac{\partial \mathcal{L}}{\partial v}(\Omega, u(\Omega), q), u^{\prime}(\Omega)(\theta)\right\rangle+\frac{\partial \mathcal{L}}{\partial \Omega}(\Omega, u(\Omega), q)(\theta) .
$$

Then taking $q=p(\Omega)$ cancels out the first term in the above right-hand side because of the adjoint equation. Therefore, the shape derivative of $J$ in the direction $\theta$ is

$$
J^{\prime}(\Omega)(\theta)=\frac{\partial \mathcal{L}}{\partial \Omega}(\Omega, u(\Omega), p(\Omega))(\theta) .
$$

Applying Lemma 3.2 and recalling that $\Gamma_{N}$ is fixed, we obtain

$$
\frac{\partial \mathcal{L}}{\partial \Omega}(\Omega, u, p)(\theta)=\int_{\Gamma} \theta \cdot n(j(\sigma)+A e(u) \cdot e(p)) d s .
$$

This proof is only a formal computation because we assume that $u$ is shape differentiable but it can be rigorously justified (see the references quoted above).

Remark 3.4. Lemma 3.2 yields the shape derivative of the volume constraint:

$$
V(\Omega)=\int_{\Omega} d x \quad \Rightarrow \quad V^{\prime}(\Omega)(\theta)=\int_{\partial \Omega} \theta \cdot n d s .
$$

\section{Topological derivative}

The notion of topological gradient (in the context of optimal design) has been introduced by Schumacher, Masmoudi, Sokolowski and their co-workers [18], [15], [19], [36], [37] and has been further developped by many authors [11], [25], [32]. The same idea has also been proposed independently in the context of inverse problems by Ammari and his co-workers [8], [9], [10]. The main idea of the topological gradient method is to test the optimality of a domain to topology variations by removing a small hole with appropriate boundary conditions. Indeed, one drawback of the previous method of shape derivative is that there is no change of topology in the parametrization $\Omega_{\theta}$. Numerical methods based on the shape derivative may therefore fall into a local minimum (corresponding to the initial topology).

We give a brief overview of the topological derivative and we refer to the above quoted references for details and proofs. For a given reference domain $\Omega \subset \mathbb{R}^{d}$, a point $x_{0} \in \Omega$ and a model hole $\omega \subset \mathbb{R}^{d}$, we define the translated and rescaled hole

$$
\omega_{\rho}=x_{0}+\rho \omega, \quad \forall \rho>0,
$$


and the perforated domain

$$
\Omega_{\rho}=\Omega \backslash \bar{\omega}_{\rho} .
$$

By insertion of a hole, the class of homotopy of $\Omega_{\rho}$ is different from that of the reference domain $\Omega$. In particular, in 2-d the number of connected components of the boundary varies. Therefore, this approach, which performs topology variations, is very different from the previous approach of shape derivative where the class of homotopy of $\Omega_{\theta}$, defined by (8), is always the same.

The objective function $J\left(\Omega_{\rho}\right)$ is computed with the elastic displacement $u_{\rho}$, solution of the elasticity problem (2) where $\Omega$ is replaced by $\Omega_{\rho}$ and homogeneous Neumann boundary conditions (no traction) are imposed on $\partial \omega_{\rho}$.

Definition 4.1. If the objective function admits the following so-called topological asymptotic expansion for small $\rho>0$

$$
J\left(\Omega_{\rho}\right)=J(\Omega)+\rho^{d} D_{T} J\left(x_{0}\right)+o\left(\rho^{d}\right),
$$

then $D_{T} J\left(x_{0}\right)$ is called the topological derivative at point $x_{0}$.

It is a simple exercise to compute the topological derivative of the volume of $\Omega, V(\Omega)=\int_{\Omega} d x$, which is simply

$$
D_{T} V(x)=-|\omega|
$$

The topological derivative of the objective function (3) is less straightforward: it has been obtained in [36], [37]. From now on, we restrict ourselves to the 2-d case and to a spherical model hole $\omega$. This simplifies greatly the computations of the topological derivatives. We do not discuss the 3 -d case since our numerical experience shows that the topological gradient is not a necessary tool for obtaining complex topologies in 3-d, even if the initial guess is the full reference domain without holes.

Theorem 4.2 ([36]). For $d=2$, take $\omega$ to be the unit ball of $\mathbb{R}^{2}$. Assume that the state $u$, solution of (2), and the adjoint state $p$, solution of (10), are smooth. We denote by $\sigma(u)$ and $\sigma(p)$ the corresponding stress tensors, with eigenvalues $\sigma_{1,2}(u)$ and $\sigma_{1,2}(p)$. For any $x \in \Omega$ the topological derivative of (3) is

$$
D_{T} J(x)=-2 \pi\left(a_{u}^{2}+2 b_{u}^{2}+\frac{a_{u} a_{p}+2 b_{u} b_{p} \cos (2 \delta)}{E}\right)(x),
$$

where $E$ is the Young's modulus, given in terms of the Lamé moduli by $E=$ $\mu(3 \lambda+2 \mu) /(\mu+\lambda)$,

$$
\begin{array}{ll}
a_{u}=\operatorname{tr} \sigma(u), & b_{u}=\sigma_{1}(u)-\sigma_{2}(u), \\
a_{p}=\operatorname{tr} \sigma(p), & b_{p}=\sigma_{1}(p)-\sigma_{2}(p),
\end{array}
$$

and $\delta$ is the angle between the eigenvectors of $\sigma(u)$ and $\sigma(p)$. 
The numerical application of the topological derivative is as follows. Consider the minimization of the Lagrangian

$$
\mathcal{L}(\Omega)=J(\Omega)+\ell|\Omega|,
$$

where $\ell$ is a given Lagrange multiplier. The corresponding topological gradient is

$$
D_{T} \mathcal{L}(x)=D_{T} J(x)-\ell|\omega| .
$$

At the points $x$ where $D_{T} \mathcal{L}(x)$ is negative, we introduce holes into the current domain $\Omega$. Since this criterion applies for infinitesimal holes, we should not remove too much material. In practice it is better to nucleate holes only at the minimum (negative) points of this topological derivative. The coupling of the topological gradient method with the level set algorithm has been studied in [3], [13], [41].

\section{Level set method and optimization algorithm}

This section recalls the framework of the level set method as proposed by Osher and Sethian [28] (see also the books [26] and [33]). The application of the level set method to structural optimization was pioneering in [6], [7], [27], [34], [40]. Consider $D \subset \mathbb{R}^{d}$ a bounded domain in which all admissible shapes $\Omega$ are included, i.e. $\Omega \subset D$. In numerical practice, the domain $D$ will be uniformly meshed once and for all. We parameterize the boundary of $\Omega$ by means of a level set function $\psi$, defined in $D$ by

$$
\left\{\begin{aligned}
\psi(x)=0 & \Leftrightarrow x \in \partial \Omega \cap D, \\
\psi(x)<0 & \Leftrightarrow x \in \Omega, \\
\psi(x)>0 & \Leftrightarrow x \in(D \backslash \bar{\Omega}) .
\end{aligned}\right.
$$

The normal $n$ to the shape $\Omega$ is recovered as $\nabla \psi /|\nabla \psi|$ and the mean curvature $H$ is given by the divergence of the normal $\operatorname{div}(\nabla \psi /|\nabla \psi|)$ (these quantities are computed throughout the whole domain $D$ and are thus natural extensions of their original definition on the boundary $\partial \Omega$ ).

During the optimization process, the shape $\Omega(t)$ is going to evolve according to a fictitious time parameter $t \in \mathbb{R}^{+}$which corresponds to descent stepping. The evolution of the level set function is governed by the following HamiltonJacobi transport equation [28]

$$
\frac{\partial \psi}{\partial t}+V|\nabla \psi|=0 \quad \text { in } \quad D,
$$

where $V(t, x)$ is the normal velocity of the shape's boundary (a scalar function from $\mathbb{R}^{+} \times D$ into $\mathbb{R}$ ). Equation (18) is simply obtained by differentiating the definition of a level set of $\psi, \psi(t, x(t))=$ Cst, and replacing the velocity $\dot{x}(t)$ by $V n$. The main advantage of the non-linear equation (18) with respect to a simpler linear transport equation (involving a vector velocity) is that every point 
$x \in D$ moves along the normal direction to the level set of $\psi$ in $x$. Recall that, in theory, a tangential velocity does not change the level sets of $\psi$, although in practice it yields numerical diffusion which may cause large errors in the position of the boundary $\partial \Omega$. Furthermore, (18) takes care of possible self-intersections of the level sets of $\psi$ and appropriately regularize, or not, possible corners in the shape (see [26], [33]).

The choice of the normal velocity $V$ is based on the shape derivative computed in Theorem 3.3 for the Lagrangian (16)

$$
\mathcal{L}^{\prime}(\Omega)(\theta)=\int_{\partial \Omega}-V \theta \cdot n d s,
$$

where the integrand $V$ is given in terms of the state $u$ and adjoint state $p$ by

$$
V=-(j(\sigma)+A e(u) \cdot e(p)+\ell)
$$

with $\ell$, the Lagrange multiplier for the volume constraint as defined in (7). Remark that $V$ is defined everywhere in $D$ and not only on the boundary $\partial \Omega$, which is a crucial point for solving (18). We have implicitly chosen a simple normal velocity based on steepest descent, $\theta=V n$. Transporting $\psi$ by (18) is equivalent to moving the boundary $\partial \Omega$ (the zero level set of $\psi$ ) along the descent gradient direction $-\mathcal{L}^{\prime}(\Omega)$. The length of the time interval on which (18) is integrated corresponds to the descent step. Other choices of the velocity are possible, corresponding to different choices of the inner product between $\mathcal{L}^{\prime}(\Omega)$ and $\theta$, or to a preconditioning of the gradient method (see [12], [20] for details). Overall, the level set method allows us to replace the Lagrangian evolution of the boundary $\partial \Omega$ by the Eulerian solution of a transport equation in the entire fixed domain $D$. On the same token, the elasticity equations for the state $u$ (and for the adjoint state $p$ ) are extended to the whole domain $D$ by using the so-called "ersatz material" approach. It amounts to fill the holes $D \backslash \Omega$ by a weak phase mimicking void but avoiding the singularity of the stiffness matrix. This is a well-known procedure in topology optimization which we already described in our previous work [7]. In numerical practice, the weak material mimicking holes in $D \backslash \Omega$ is chosen as $10^{-3} \mathrm{~A}$.

It is possible to couple this level set algorithm with the topological derivative $D_{T} \mathcal{L}(x)$ of Section 4 as follows. In a first step, the level set function $\psi$ is advected according to the velocity $V$ by solving (18). In a second step, holes are introduced into the current domain $\Omega$ where the topological derivative $D_{T} \mathcal{L}(x)$ is minimum and negative. More precisely, at those points we change the negative sign of the level set function $\psi$ into a positive sign, according to the parametrization (17). A reinitialization is then performed in order to smooth the resulting level set function (see [3]).

In practice, it is better to perform more level set steps than topological gradient steps. Therefore, the main parameter of our coupled algorithm is an integer $n_{\text {opt }}$ which is the number of gradient steps between two successive application of the topological gradient. The value of $n_{\text {opt }}$ is given for each example in Section 6. Sometimes we simply take $n_{\text {opt }}=+\infty$ which means we 
do not use at all the topological derivative (for instance, this is the case for 3-d computations). Our proposed algorithm for the minimization of (6) with the shape derivative (9) and the topological derivative (14) is an iterative method, structured as follows:

1. Initialization of the level set function $\psi_{0}$ corresponding to an initial guess $\Omega_{0}$.

2. Iteration until convergence, for $k \geq 0$ :

(a) Elasticity analysis. Computation of the state $u_{k}$ and adjoint state $p_{k}$ through two problems of linear elasticity posed in $\Omega_{k}$. This yields the values of the shape derivative and of the topological gradient.

(b) Shape gradient. If $\bmod \left(k, n_{\text {top }}\right)<n_{\text {top }}$, the current shape $\Omega_{k}$, characterized by the level set function $\psi_{k}$, is deformed into a new shape $\Omega_{k+1}$, characterized by $\psi_{k+1}$ which is the solution of the transport Hamilton-Jacobi equation (18) after a time interval $\Delta t_{k}$ with the initial condition $\psi_{k}$ and a velocity $V_{k}$ computed in terms of $u_{k}$ and $p_{k}$. The time of integration $\Delta t_{k}$ is chosen such that $\mathcal{L}\left(\Omega_{k+1}\right) \leq \mathcal{L}\left(\Omega_{k}\right)$.

(c) Topological gradient. If $\bmod \left(k, n_{\text {top }}\right)=0$, we perform a nucleation step. We obtain a new shape $\Omega_{k+1}$ by inserting new holes into the current shape $\Omega_{k}$. Namely, the sign of the level set function $\psi_{k}$ is changed from negative to positive values where the topological derivative $D_{T} \mathcal{L}_{k}$, depending on $u_{k}$ and $p_{k}$, has minimum negative values. If the objective function has increased, i.e. if $\mathcal{L}\left(\Omega_{k+1}\right)>\mathcal{L}\left(\Omega_{k}\right)$, then no holes are nucleated and we just take $\Omega_{k+1}=\Omega_{k}$.

The Hamilton-Jacobi equation (18) is solved by a finite difference, explicit, second order, upwind scheme (see [26], [33]) on a Cartesian grid (we also implemented a finite volume type scheme on unstructured meshes, see [4]). The boundary conditions for $\psi$ are of Neumann type. Since this scheme is explicit in time, its time stepping must satisfy a CFL condition (which implies that the boundary $\partial \Omega$ can not move from more than one cell at each time step). However, a finite element analysis being much more computationally intensive, the integration time $\Delta t_{k}$ at iteration $k$ in the above algorithm is not limited to a single CFL time step but is usually equal to roughly 20 to 50 of those explicit time steps. We thus perform several explicit time steps for (18) before re-evaluating the shape derivative, i.e., computing the state $u$ and adjoint $p$. The precise number of time steps is controlled by the decrease of the objective function.

The Lagrange multiplier $\ell_{k}$ for the volume constraint is updated at each iteration by increasing or decreasing its values if the actual volume is larger or smaller than the target volume in (5).

In order to regularize the level set function (which may become too steep), we reinitialize it periodically by solving another Hamilton-Jacobi equation which admits as a stationary solution the signed distance to the initial interface [33]. For details of numerical implementation we refer to [7]. 


\section{$6 \quad$ Numerical results}

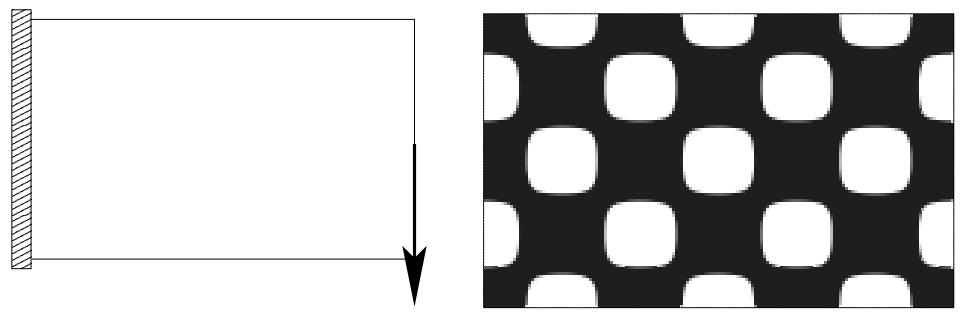

Figure 1: Boundary conditions and initialization for the cantilever problem.

In all examples below the Young modulus is 1 , the Poisson ratio is 0.3 , the mesh is uniformly rectangular with $Q 1$ finite elements for the displacement and a second-order explicit upwind finite difference scheme for the Hamilton-Jacobi equation. We perform of the order of 10 explicit time steps for the HamiltonJacobi equation for each elasticity analysis (that we call iteration).

\begin{tabular}{|l||c|c|c|}
\hline Optimal design for: & Compliance & $\int|\sigma|^{2}$ & $\int|\sigma|^{5}$ \\
\hline \hline value of the compliance & $\mathbf{7 8 . 1 9 5}$ & 78.305 & 79.620 \\
\hline value of $\int|\sigma|^{2}$ & 82.574 & $\mathbf{8 2 . 5 1 3}$ & 84.140 \\
\hline value of $\int|\sigma|^{5}$ & $5.367 \mathrm{E}+5$ & $5.434 \mathrm{E}+5$ & $\mathbf{4 . 7 5 4 E + 5}$ \\
\hline
\end{tabular}

Table 1: Values of the objective functions for the three optimal designs of Figure 2 .

\section{Cantilever:}

Our first test case is a classical 2-d cantilever problem (see Figure 6). The size of the domain is $1.6 \times 1$. discretized by $160 \times 100$ square cells. We start with an initialization with many holes and we do not use the topological gradient (i.e., $\left.n_{\text {opt }}=+\infty\right)$. We compare the optimal shapes and topologies for three different objective functions

$$
\int_{\Omega} A^{-1} \sigma \cdot \sigma d x, \quad \int_{\Omega}|\sigma|^{2} d x, \quad \int_{\Omega}|\sigma|^{5} d x,
$$

the first one being the usual compliance. Note that the topological derivative is explicitly known for the two first objective functions, but not for the third one. With the same initialization the optimal topologies are the same and the shapes are only slightly different. We display on Figure 2 the optimal shapes and a zoom near the tip of the cantilever where one can see some minor differences. 
One possible explanation for these similar optimal designs is that the compliance optimality condition (see [7]) states that the boundary is iso-stressed: there are therefore no stress concentrations at the corners of the truss structure (because the bars are optimally oriented) except where the load is applied. We check on Table 6 that our optimal designs are actually optimal for their corresponding objective functions and not for the other ones! The convergence is obtained in less than 200 iterations as can be checked on Figure 3.

One advantage of the level set method is that we always obtain "black and white designs" with no composite areas or possible checkerboard instabilities as is sometimes the case with the SIMP or homogenization methods. In particular, we do not encounter the so-called stress singularity problem as described in $[1],[5],[16],[30]$ (which is very sensitive in the penalization process of the homogenization method).
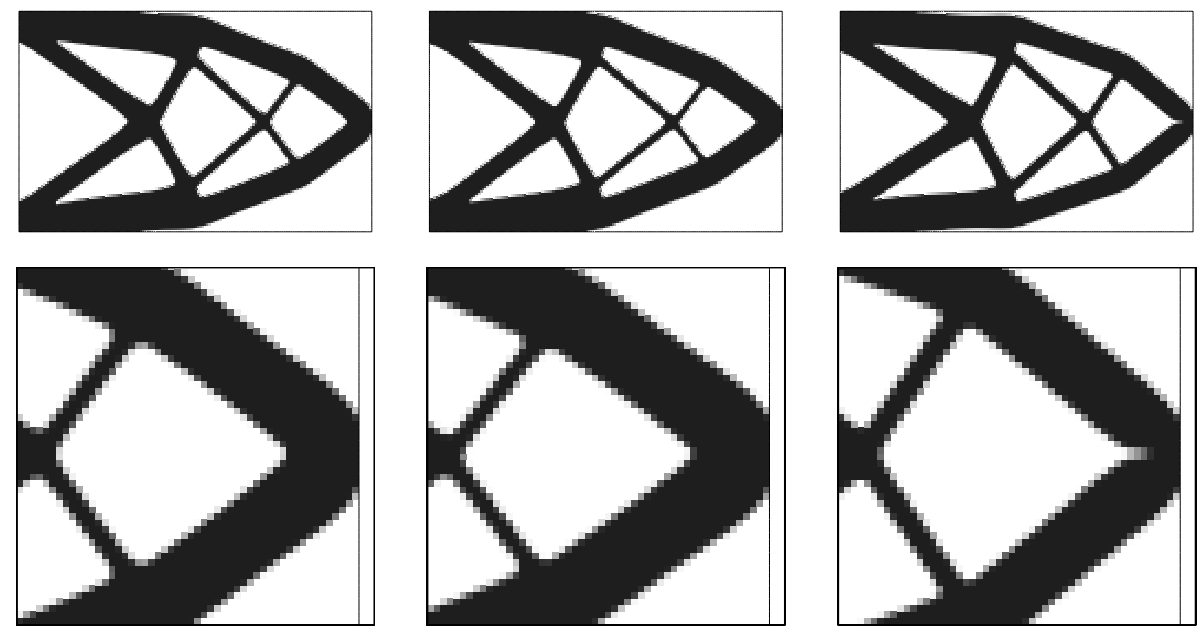

Figure 2: Optimal cantilevers for the compliance (left), $\int|\sigma|^{2}$ (middle) and $\int|\sigma|^{5}$ (right). The bottom line is a zoom near the tip of the cantilever.

We revisit the previous cantilever problem with an initialization which is the full domain $D$, using the topological gradient with $n_{o p t}=5$. We minimize the objective function $\int_{\Omega}|\sigma|^{2} d x$. As can be checked on Figure 4 we obtain the same shape and topology as in Figure 2 (middle). However the present computation was not relying on any clever initial guess.

\section{L-beam:}

Our second test case is the L-beam problem already studied e.g. in [5]. On Figure 5 we display the boundary conditions and the optimal designs for three objective functions of the type

$$
\int_{\Omega} k(x)|\sigma|^{\alpha} d x, \quad \text { with } \alpha=2,5,10 .
$$




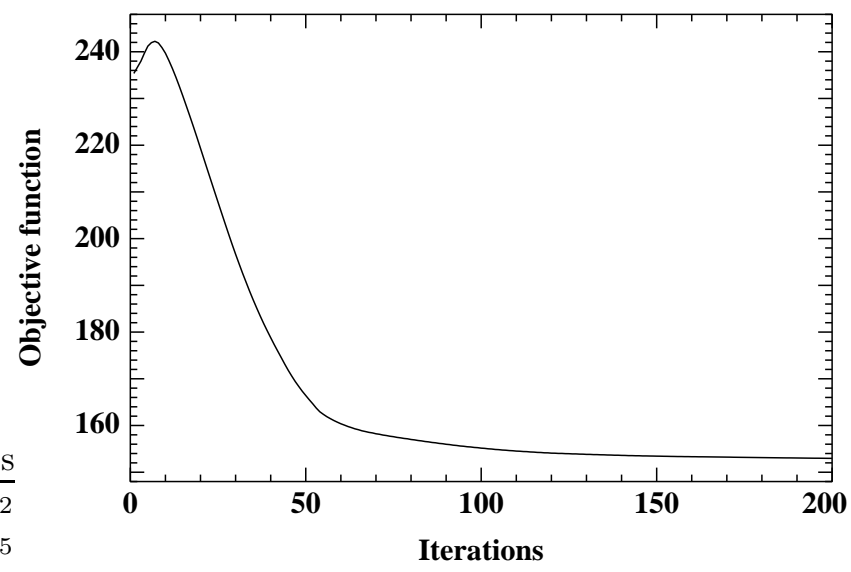

Figure 3: Typical convergence history of the algorithm for $\int|\sigma|^{5}$.

The localizing function $k(x)$ is equal to 1 everywhere except on a small zone around the point on the right side where the vertical load is applied, where it is set to 0 . We use such a localizing function because our goal is to reduce the stress singularity developed in the re-entrant corner and not the one caused by the applied load. We do not use the topological gradient (i.e., $n_{\text {opt }}=+\infty$ ). In this case the three optimal designs are quite different. In particular, for $\alpha=10$ we clearly see that the shape is smoothed and "rounded" around the re-entrant corner where a stress singularity can develop.

\section{Gripping mechanism:}

Our third test case is a gripping mechanism design problem [7], [35]. The goal is to design a compliant mechanism such that, if an horizontal load is applied to the left side, the jaws on the right side close. The jaws are the black zones on Figure 6. The domain is fixed on the lower and upper part of its left boundary. We optimize the structure using two different objective functions. The first one is the "geometrical advantage" (GA), widely used for the compliant mechanism design (see e.g. [35]). It consists in maximizing the ratio $u_{\text {out }} / u_{\text {in }}$ where $u_{\text {out }}$ is the mean displacement of the jaws in the direction $e_{2}$ (the vertical unit vector) and $u_{i n}$ is the mean displacement on the zone submitted to the input force (see [22] for details). The second objective function is

$$
\int_{\Omega} k(x)\left|\sigma-\sigma_{0}(x)\right|^{2} d x,
$$

where, denoting by $Z$ the black zone of the jaws,

$$
k(x)=\left\{\begin{array}{ll}
1 & \text { in } Z \\
0.1 & \text { in } \Omega \backslash Z
\end{array}, \quad \sigma_{0}(x)=\left\{\begin{array}{ll} 
\pm e_{2} \otimes e_{2} & \text { in } Z \\
0 & \text { in } \Omega \backslash Z
\end{array},\right.\right.
$$



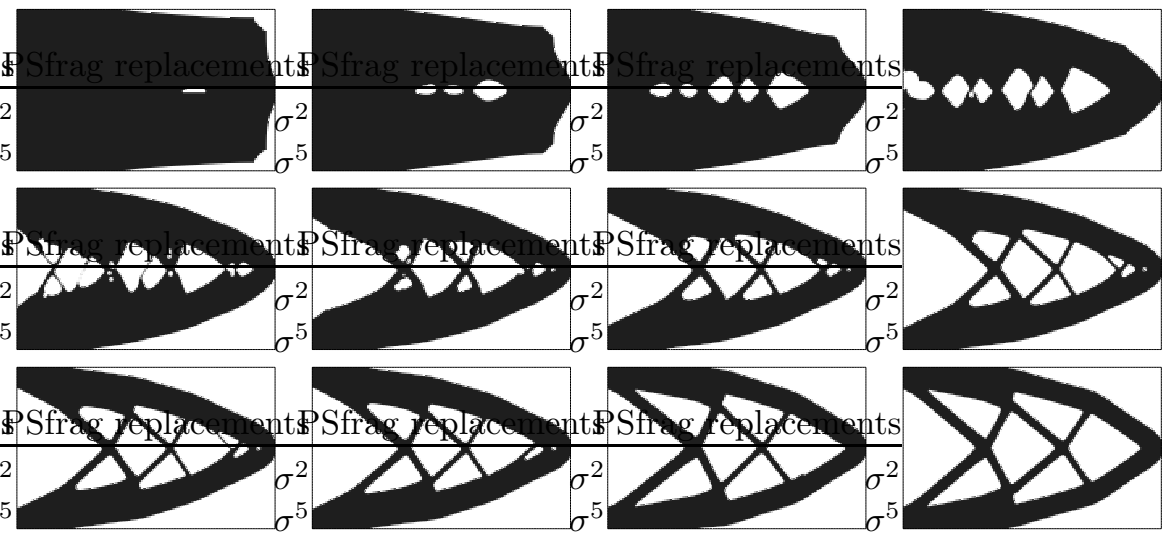

Figure 4: Topological gradient: successive configurations obtained by the numerical algorithm coupled with the topological gradient. One evaluation of the topological gradient is made every 5 iterations up to iteration number 100 (snapshots at iterations 10, 20, 30, 40, 50, 60, 70, 80, 90, 100, 200 and 300)

where $\sigma_{0}$ takes opposite values on the opposite faces of the jaws. These values have been chosen to allow a good strength on the jaws together with a control on the stresses over the whole structure. No volume constraint is imposed on both objective functions.

When the structure is optimized for the GA, the jaws support a traction free (homogeneous Neumann) boundary condition, while they are fixed (Dirichlet boundary condition) for the optimization of the stress criterion. Thus, the obtained optimal structures are quite different: the GA leads to very compliant mechanisms, with many weak hinges. The second objective function optimizes stiffer mechanisms, with less displacement of the jaws but more tightening strength. The optimal configurations for both objective functions are displayed on Figure 7, with the deformed configurations. We did not use the topological gradient.

To evaluate the performances of the gripping mechanisms optimized for the two objective functions we compute, for each structure, its behavior when the external force is applied with and without blocking the jaws. Figure 8 shows the distribution of the maximal principal stress on each gripping mechanism in both configuration. The grayscale corresponds to the same stress values on the four plots. It is obvious that the global stress level is lower in the structure optimized with the stress objective function than in the other one.

\section{A 3d case:}

Our fourth and last test case is a 3-d optimal mast. The boundary conditions are displayed on Figure 9. The four corners of the bottom of the structure are fixed. By symmetry the computation is done on one fourth of the structure. The optimal designs for the compliance and for $\int_{\Omega}|\sigma|^{2} d x$ are shown on Figure 10 


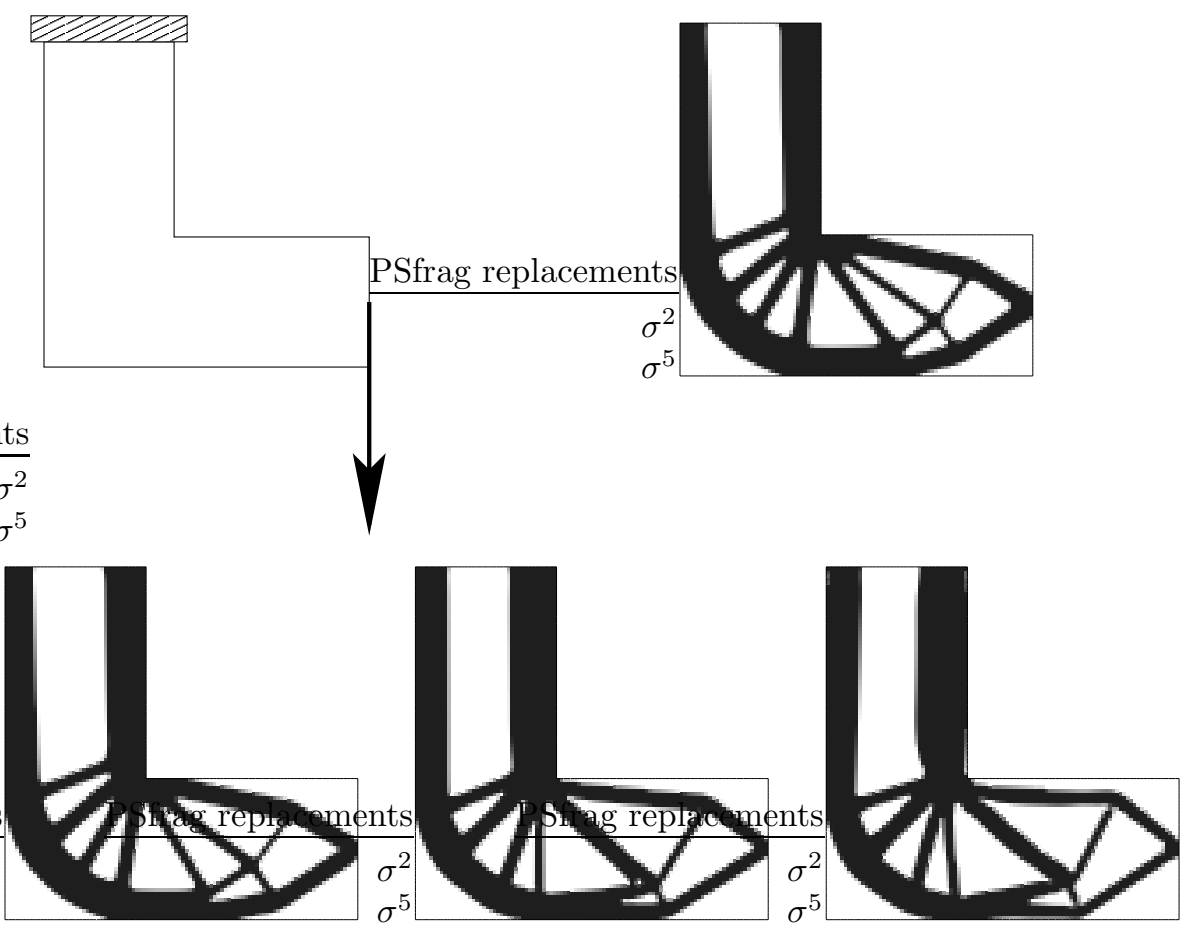

Figure 5: L-beam problem. Top: boundary conditions and optimal structure for the compliance. Bottom: optimal structures for $\int|\sigma|^{\alpha}$ with $\alpha=2$ (left), 5 (middle) and 10 (right).

where one can see the efficiency of the level set method for capturing complex topologies. No topological gradient is used here. According to our experience it is not a necessary ingredient: different initializations yield the same results.

\section{ACKNOWLEDGMENTS}

This work was partly supported by the MULTIMAT european network MRTN-CT-2004-505226 funded by the EEC. 


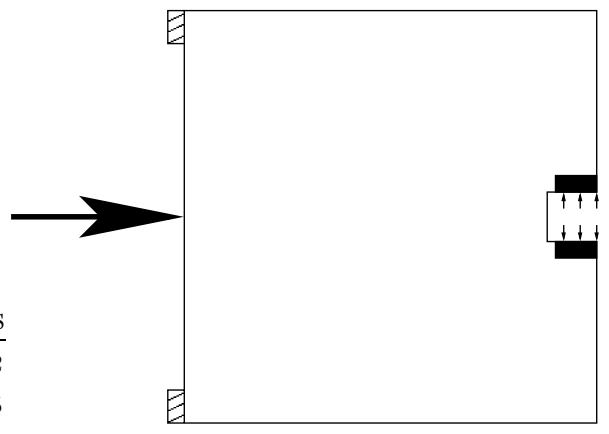

Figure 6: Boundary conditions for the gripping mechanism.
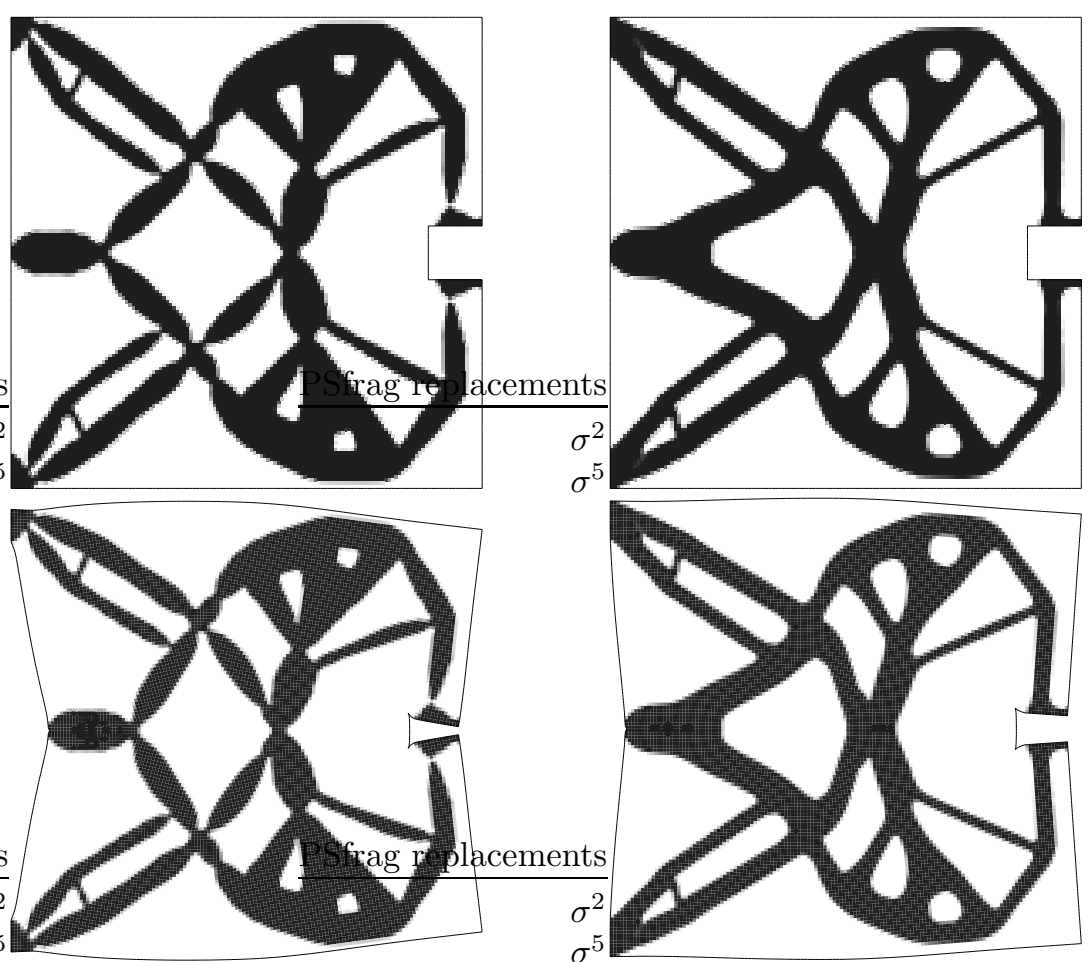

Figure 7: Gripping mechanism. Top: optimal designs for the "geometrical advantage" GA (left) and the stress criterion (right). Bottom: deformed configuration of both structures. 

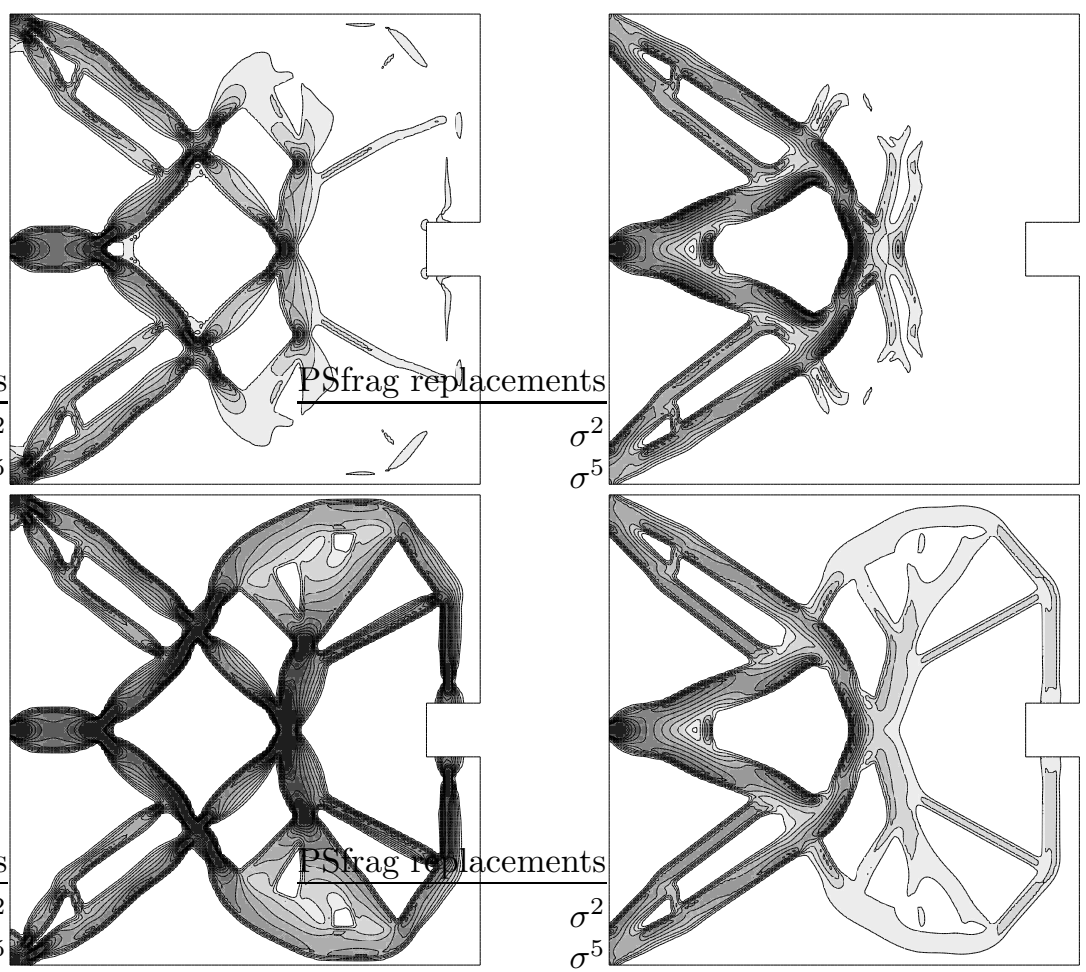

Figure 8: Stress distribution in the gripping mechanisms (left: optimized for GA, right: optimized for the stress criterion) with two different boundary conditions: top: jaws free, bottom: jaws clamped.

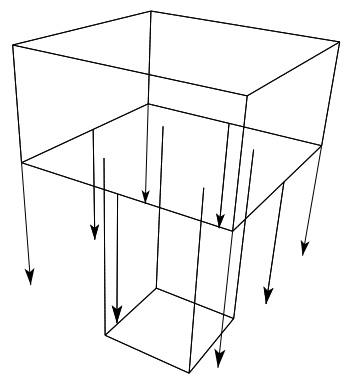

Figure 9: Boundary conditions for the 3-d optimal mast. 

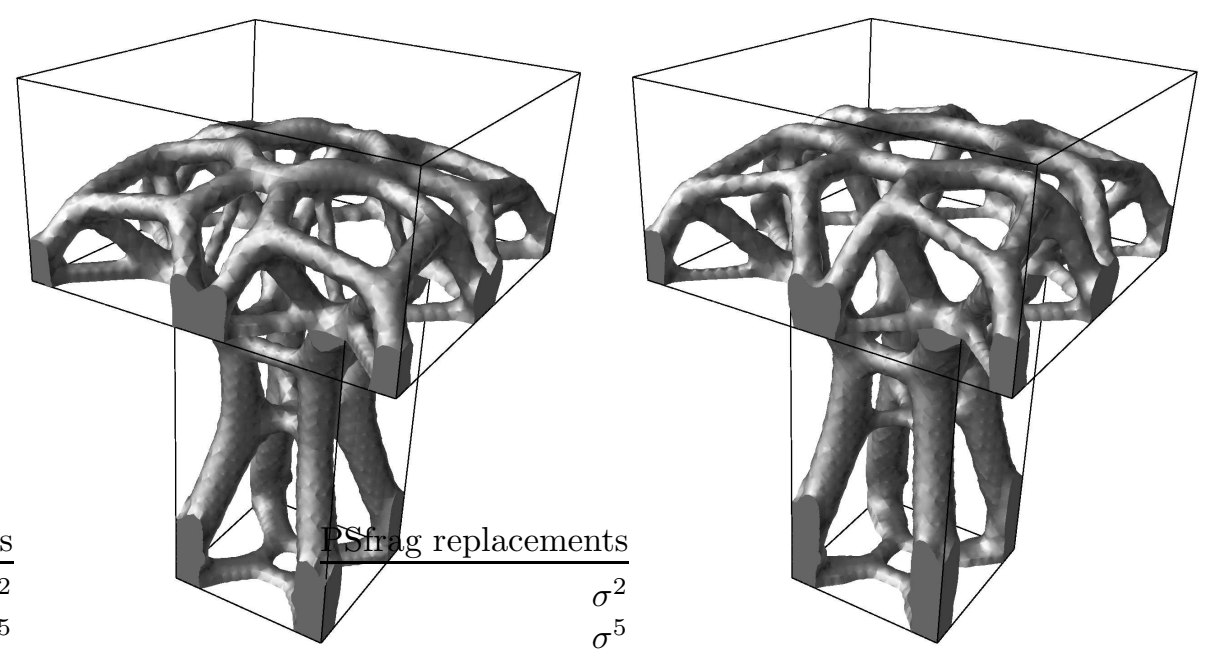

Figure 10: Optimal 3-d masts for the compliance (left), and $\int|\sigma|^{2}$ (right). 


\section{References}

[1] Achtziger W., Topology Optimization Subject to Design-Dependent Validity of Constraints, in Topology optimization of structures and composite continua, G.I.N. Rozvany and N. Olhoff eds., 177-191, Kluwer Academic Publishers (2000).

[2] Allaire, G., Conception optimale de structures, Mathématiques \& Applications, 58, Springer Verlag, Heidelberg (2006).

[3] Allaire G., Jouve F., de Gournay F., Toader A.-M., Structural optimization using topological and shape sensitivity via a level set method, Control and Cybernetics 34, 59-80 (2005).

[4] Allaire G., Jouve F., A level-set method for vibration and multiple loads structural optimization, Comput. Methods Appl. Mech. Engrg. 194, 3269$3290(2005)$.

[5] Allaire G., Jouve F., Maillot H., Topology optimization for minimum stress design with the homogenization method, Structural and Multidisciplinary Optimization, 28, 87-98 (2004).

[6] Allaire G., Jouve F., Toader A.-M., A level set method for shape optimization, C. R. Acad. Sci. Paris, Série I, 334, 1125-1130 (2002).

[7] Allaire G., Jouve F., Toader A.-M., Structural optimization using sensitivity analysis and a level set method, J. Comp. Phys., Vol 194/1, 363-393 (2004).

[8] Ammari H., Kang H., Reconstruction of small inhomogeneities from boundary measurements, Lecture Notes in Mathematics, 1846. Springer-Verlag, Berlin, (2004).

[9] Ammari H., Kang H., Nakamura G., Tanuma K., Complete asymptotic expansions of solutions of the system of elastostatics in the presence of an inclusion of small diameter and detection of an inclusion, J. Elasticity 67 , no. 2, 97-129 (2002).

[10] Ammari H., Vogelius M., Volkov D., Asymptotic formulas for perturbations in the electromagnetic fields due to the presence of inhomogeneities of small diameter. II. The full Maxwell equations, J. Math. Pures Appl. (9) 80, no. 8, 769-814 (2001).

[11] Amstutz S., Topological sensitivity analysis for some nonlinear PDE system, J. Math. Pures Appl. (9) 85, no. 4, 540-557 (2006).

[12] Burger M., A framework for the construction of level set methods for shape optimization and reconstruction, Interfaces and Free Boundaries, 5, 301329 (2003). 
[13] Burger M., Hackl B., Ring W., Incorporating topological derivatives into level set methods, J. Comp. Phys., Vol 194/1, 344-362 (2004).

[14] Céa J., Conception optimale ou identification de formes, calcul rapide de la dérivée directionnelle de la fonction coût, Math. Model. Num. Anal. 20, 3, 371-402 (1986).

[15] Céa J., Garreau S., Guillaume P., Masmoudi M., The shape and topological optimizations connection, IV WCCM, Part II (Buenos Aires, 1998), Comput. Methods Appl. Mech. Engrg. 188, 713-726 (2000).

[16] Cheng G.D., Guo X., Study on topology optimization with stress constraints, Eng. Optim. 20, pp.129-148 (1992).

[17] Duysinx P., Bendsoe M., Topology Optimization of Continuum Structures with Local Stress Constraints, Int. J. Num. Meth. Engng., 43, 1453-1478 (1998).

[18] Eschenauer H., Kobelev V., Schumacher A., Bubble method for topology and shape optimization of structures, Structural Optimization, 8, 42-51 (1994).

[19] Garreau S., Guillaume P., Masmoudi M., The topological asymptotic for PDE systems: the elasticity case. SIAM J. Control Optim. 39, no. 6, 17561778 (2001).

[20] de Gournay F., Velocity extension for the level-set method and multiple eigenvalues in shape optimization. SIAM J. on Control and Optim., 45, no. 1, 343-367 (2006).

[21] de Gournay F., Allaire G., Jouve F., Shape and topology optimization of the robust compliance via the level set method, to appear in COCV (2007).

[22] Jouve F., Mechkour H., Optimal design of compliant mechanisms using the level set method, preprint, (2006).

[23] Lipton, R., Design of functionally graded composite structures in the presence of stress constraints, Int. J. Solids Structures, 39, 2575-2586 (2002).

[24] Murat F., Simon S., Etudes de problèmes d'optimal design. Lecture Notes in Computer Science, 41, 54-62, Springer Verlag, Berlin (1976).

[25] Novotny A., Feijóo R., Taroco E., Padra C., Topological sensitivity analysis, Comput. Methods Appl. Mech. Engrg. 192, no. 7-8, 803-829 (2003).

[26] Osher S., Fedkiw R., Level set methods and dynamic implicit surfaces, Applied Mathematical Sciences, 153, Springer-Verlag, New York (2003).

[27] Osher S., Santosa F., Level set methods for optimization problems involving geometry and constraints: frequencies of a two-density inhomogeneous drum. J. Comp. Phys., 171, 272-288 (2001). 
[28] Osher S., Sethian J.A., Front propagating with curvature dependent speed: algorithms based on Hamilton-Jacobi formulations. J. Comp. Phys., 78, 12-49 (1988).

[29] Pedregal, P., Fully explicit quasiconvexification of the mean-square deviation of the gradient of the state in optimal design, Electr. Res. Announc. of the A.M.S., 7, 72-78 (2001).

[30] Pereira J.T., Fancello E.A., Barcellos C.S., Topology optimization of continuum structures with material failure constraints, Struct. Multidisc. Optim. 26, 50-66 (2004).

[31] Pironneau O., Optimal shape design for elliptic systems, Springer-Verlag, New York, (1984).

[32] Pommier J., Samet B., The topological asymptotic for the Helmholtz equation with Dirichlet condition on the boundary of an arbitrarily shaped hole, SIAM J. Control Optim. 43, no. 3, 899-921 (2004).

[33] Sethian J.A., Level set Methods and fast marching methods: evolving interfaces in computational geometry, fluid mechanics, computer vision and materials science, Cambridge University Press (1999).

[34] Sethian J., Wiegmann A., Structural boundary design via level set and immersed interface methods. J. Comp. Phys., 163, 489-528 (2000).

[35] Sigmund O., On the design of compliant mechanisms using topology optimization, Mech. Struct. Mach. 25, 493-524 (1997).

[36] Sokołowski J., Żochowski A., On the topological derivative in shape optimization, SIAM J. Control Optim., 37, 1251-1272 (1999).

[37] Sokołowski J., Żochowski A., Topological derivatives of shape functionals for elasticity systems. Mech. Structures Mach., 29, no. 3, 331-349 (2001).

[38] Sokołowski J., Zolesio J.P., Introduction to shape optimization: shape sensitivity analysis, Springer Series in Computational Mathematics, Vol. 10, Springer, Berlin, (1992).

[39] Stainko R., Burger M., A one-shot approach to topology optimization with stress constraints, in: M.P.Bendsoe, N.Olhoff, O.Sigmund, IUTAM Symposium on Topological Design Optimization of Structures, Machines, and Materials, Springer, (2006).

[40] Wang M.Y., Wang X., Guo D., A level set method for structural topology optimization, Comput. Methods Appl. Mech. Engrg., 192, 227-246 (2003).

[41] Wang X., Yulin M., Wang M.Y., Incorporating topological derivatives into level set methods for structural topology optimization, in Optimal shape design and modeling, T. Lewinski et al. eds., 145-157, Polish Academy of Sciences, Warsaw (2004). 